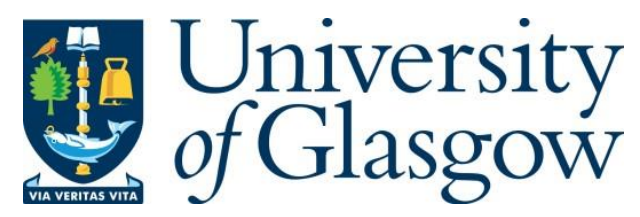

Huang, Y., Karadimas, P. and Pour Sohrab, A. (2019) Optimal Antenna Array Topologies for Energy Efficiency Maximization by Employing Particle Swarm Optimization. In: 2019 IEEE-APS Topical Conference on Antennas and Propagation in Wireless Communications (APWC), Granada, Spain, 09-13 Sep 2019, pp. 187-191. ISBN 9781728105673.

There may be differences between this version and the published version. You are advised to consult the publisher's version if you wish to cite from it.

$\underline{\text { http://eprints.gla.ac.uk/227360/ }}$

Deposited on: 8 January 2021

Enlighten - Research publications by members of the University of Glasgow http://eprints.gla.ac.uk 


\title{
Optimal Antenna Array Topologies for Energy Efficiency Maximization by Employing Particle Swarm Optimization
}

\author{
Yingke Huang*, Petros Karadimas, Abed Pour Sohrab \\ School of Engineering \\ University of Glasgow \\ Glasgow, UK \\ y.huang.2@research.gla.ac.uk, Petros.Karadimas@Glasgow.ac.uk, Abed.PourSohrab@Glasgow.ac.uk
}

\begin{abstract}
The purpose of this work is to design novel antenna array topologies that maximize the energy efficiency (EE) in multiple-input-multiple-output (MIMO) wireless systems. To provide insights into EE optimization issues, the particle swarm optimization (PSO) algorithm is employed as an effective technique. First, we adopt a flexible and generalized three-dimensional (3D) angle of arrival (AOA) model to account for realistic propagation environments. Then we derive a novel closed-form spatial correlation function (SCF) relevant to the locations of antenna array elements in the 3D space. In this paper, we incorporate our SCF into the EE derivation and apply the PSO algorithm to find the optimal antenna array topology in space that maximizes the EE. The derived EE is significantly higher compared to existing stateof-the art research that employed an exponential SCF. We provide new insights into EE maximization for antenna arrays, where the simulation results demonstrate the effectiveness and usefulness of the proposed approach.
\end{abstract}

Keywords-Angle of arrival model, Antenna array topologies, energy efficiency, particle swarm optimization, spatial correlation function.

\section{INTRODUCTION}

In existing and future wireless networks, antenna arrays play an important role for channel capacity and received signal quality enhancement. However, using multiple-inputmultiple-output (MIMO) antenna arrays will also lead to increased energy consumption in terms of signal processing and extra circuitry [1]. Since the massive energy consumption becomes a global concern nowadays, investigating innovative techniques to improve the $\mathrm{EE}$ in wireless systems is of great importance [2].

Numerous works such as [3], [4] define energy efficiency (EE) with relation to channel capacity incorporating the spatial correlation function (SCF). However, the exponential SCF is widely used for simplicity in energy efficiency mathematical derivations, e.g., [1]. This simple SCF is of mathematical tractability only and cannot accurately incorporate scenarios of actual antenna array topologies operating in realistic wireless propagation environments. Besides, regardless of the locations of array elements, other existing EE maximization works are accomplished by considering the number of antenna array elements [5], signalto-noise ratio (SNR) [3], the iterations of algorithm [6], etc. To the best of our knowledge, the optimization of antenna array topologies in 3-D space to yield maximum EE has never been addressed in published literature.

In this paper, we adopt a flexible and generalized three-

This work was supported by the Engineering and Physical Sciences Research Council (EPSRC) under grant EP/R027641/1: Bandwidth and Energy Efficient Compact Multi-Antenna Systems for Connected Autonomous Vehicles. dimensional (3D) angle of arrival (AOA) model to account for realistic propagation environments. We then derive a novel closed-form SCF relevant to the locations of antenna array elements in the 3D space. The upper bounded EE and SCF can be related by combining eqs. (11) and (14) of [1]. With this upper bounded EE, we apply the particle swarm optimization (PSO) algorithm to find the optimal antenna array topologies in space for EE maximization. The EE and capacity performance when employing our modeling approach is compared with that in [1] showing significant improvement.

\section{ARRAY GEOMETRY AND ANGLE OF ARRIVAL}

\section{A. Array Geometry}

For illustration, there are two antenna array elements with corresponding locations in a 3D spatial coordinate $(X, Y, Z)$ as shown in Fig. 1. The location of the $m$ th element in space with the phase reference at the origin is referred by $\left(x_{m}, y_{m}, z_{m}\right)$, and the associated position vector can be denoted by $\mathbf{r}_{m}=\left(x_{m} y_{m} z_{m}\right)^{T}$. As illustrated in Fig. 1, $\theta$ and $\varphi$ indicate the elevation AOA and azimuth AOA, respectively, and satisfy $\theta \in\left[-\frac{\pi}{2}, \frac{\pi}{2}\right], \varphi \in[-\pi, \pi]$. Due to the definitions of $\theta$ and $\varphi$, the wave vector can be expressed as [7]

$$
\mathbf{k}(\theta, \varphi)=\frac{2 \pi}{\lambda}\left[\begin{array}{c}
\cos (\theta) \cos (\varphi) \\
\cos (\theta) \sin (\varphi) \\
\sin (\theta)
\end{array}\right]
$$

Let $j=\sqrt{-1}$, the $m$ th input of the steering vector, i.e., the phase delay of a multipath component with the AOA $(\theta, \varphi)$ impinging on the $m$ th array element is given by [7]

$$
\begin{aligned}
& v_{m}(\theta, \varphi)=\exp \left\{-j\left[\mathbf{r}_{m} \cdot \mathbf{k}(\theta, \varphi)\right]\right\}= \\
& \exp \left\{-j(2 \pi / \lambda)\left[x_{m} \cos (\theta) \cos (\varphi)+\right.\right. \\
& \left.\left.y_{m} \cos (\theta) \sin (\varphi)+z_{m} \sin (\theta)\right]\right\} .
\end{aligned}
$$

Slightly different from the wave vector, the difference vector between $\mathbf{r}_{m}$ and $\mathbf{r}_{n}$ is expressed as 


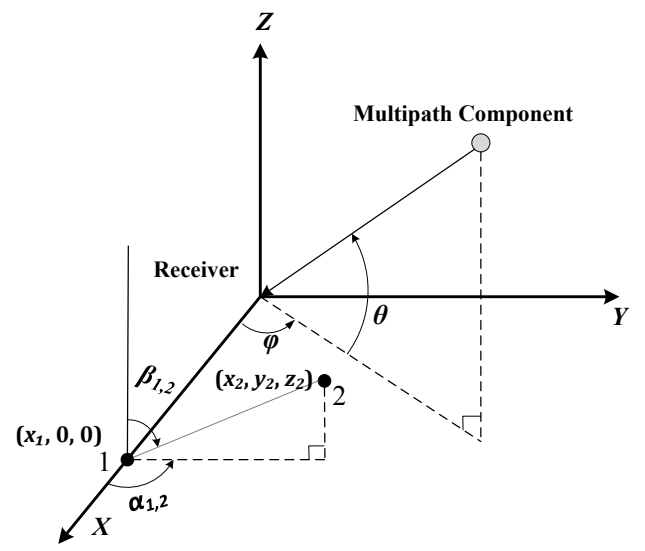

Fig. 1. A 3-D coordinate system with 4 array elements in space.

$$
\mathbf{d}_{m, n}=\mathbf{r}_{m}-\mathbf{r}_{n}=\left[\begin{array}{c}
r_{m, n} \sin \left(\beta_{m, n}\right) \cos \left(\alpha_{m, n}\right) \\
r_{m, n} \sin \left(\beta_{m, n}\right) \sin \left(\alpha_{m, n}\right) \\
r_{m, n} \cos \left(\beta_{m, n}\right)
\end{array}\right]
$$

where $r_{m, n}=\sqrt{\left(x_{m}-x_{n}\right)^{2}+\left(y_{m}-y_{n}\right)^{2}+\left(z_{m}-z_{n}\right)^{2}}, \beta_{m, n}$ and $\alpha_{m, n}$ denote the angle between $\mathbf{d}_{m, n}$ and the $\mathrm{Z}$ axis and the angle between $\mathbf{d}_{m, n}$ and the $\mathrm{X}$ axis, respectively (see Fig. 1).

\section{B. Angle of Arrival}

We employ the Gaussian distribution for both the azimuth and elevation AOA as a generic model that can incorporate directional propagation [8]. Assuming they are independent to each other, the joint AOA probability density function (PDF), $P(\theta, \varphi)$, can be written as $P(\theta, \varphi)=P_{\theta}(\theta) P_{\varphi}(\varphi)$, where $P_{\theta}(\theta)$ and $P_{\varphi}(\varphi)$ are the marginal PDFs for the elevation and azimuth AOA, respectively. Thus [8]

$$
\begin{aligned}
P(\theta, \varphi)=\frac{C_{\theta}}{\sqrt{2 \pi} \Delta \theta} \exp \left[-\frac{\left(\theta-\theta_{0}\right)^{2}}{2 \Delta \theta^{2}}\right] \times & \frac{C_{\varphi}}{\sqrt{2 \pi} \Delta \varphi} \exp \left[-\frac{\left(\varphi-\varphi_{0}\right)^{2}}{2 \Delta \varphi^{2}}\right]
\end{aligned}
$$

where $\Delta \theta$ and $\Delta \varphi$ are the elevation angular spread (ES) and azimuth angular spread (AS), respectively. $\theta_{0}$ and $\varphi_{0}$ denote the mean elevation AOA (MEOA) and mean azimuth AOA (MAOA), respectively. Moreover, $C_{\theta}$ and $C_{\varphi}$ are the normalization factors to guarantee that $P(\theta, \varphi)$ is a PDF.

\section{MIMO CHANNEL MODEL}

\section{A. Spatial Correlation}

The SCF between the $m$ th and the $n$th array elements is given by [9]

$$
R_{s}(m, n)=E\left\{v_{m}(\theta, \varphi) v_{n}(\theta, \varphi)^{*}\right\}=
$$

$$
\int_{\theta} \int_{\varphi} v_{m}(\theta, \varphi) v_{n}(\theta, \varphi)^{*} P(\theta, \varphi) \cos (\theta) d \varphi d \theta
$$

where $(.)^{*}$ denotes the conjugate transpose. The term $\cos (\theta)$ appears in (5) due to the definition of the elevation AOS (see Fig. 1). Using eqs. (1) - (4), we can expand the SCF of (5) as

$$
\begin{aligned}
& R_{s}(m, n)= \\
& \int_{\theta} \int_{\varphi} \frac{C_{\theta} C_{\varphi}}{2 \pi \sigma_{\theta} \sigma_{\varphi}} \exp \left[-\frac{\left(\theta-\theta_{0}\right)^{2}}{2 \sigma_{\theta}^{2}}\right] \exp \left[-\frac{\left(\varphi-\varphi_{0}\right)^{2}}{2 \sigma_{\varphi}^{2}}\right] \times \\
& \exp \left\{j \frac { 2 \pi } { \lambda } r _ { m , n } \left[\sin \left(\beta_{m, n}\right) \cos (\theta) \cos \left(\alpha_{m, n}-\varphi\right)+\right.\right. \\
& \left.\left.\cos \left(\beta_{m, n}\right) \sin (\theta)\right]\right\} \cos (\theta) d \varphi d \theta .
\end{aligned}
$$

To avoid complicated numerical integration, we resort to Gauss-Hermite Quadrature (GHQ) to approximate the spatial correlation in (6) [8]. The proof is omitted here due to space limitations. The approximation is as follows

$$
\begin{aligned}
& R_{s}(m, n) \approx \frac{C_{\theta}}{\sqrt{\pi}} \cdot \frac{C_{\varphi}}{\sqrt{\pi}} \times \\
& \sum_{q=1}^{M}\left\{\omega_{q} \cdot \exp \left[j \frac{2 \pi}{\lambda} r_{m, n} \cos \left(\beta_{m, n}\right) \times \sin \left(\sqrt{2} \Delta \theta \cdot x_{q}+\theta_{0}\right)\right] \times\right. \\
& \cos \left(\sqrt{2} \Delta \theta \cdot x_{q}+\theta_{0}\right) \times \sum_{p=1}^{N}\left(\omega _ { p } \cdot \operatorname { e x p } \left[j \frac{2 \pi}{\lambda} r_{m, n} \sin \left(\beta_{m, n}\right) \times\right.\right. \\
& \left.\left.\left.\cos \left(\sqrt{2} \Delta \theta \cdot x_{q}+\theta_{0}\right) \times \cos \left(\alpha_{m, n}-\sqrt{2} \Delta \varphi \cdot x_{p}-\varphi_{0}\right)\right]\right)\right\}
\end{aligned}
$$

where the $x_{q}(q=1,2, \ldots M), x_{p}(p=1,2, \ldots, N)$ are the roots of corresponding $M$-th and $N$-th order Hermite polynomials, and $\omega_{q}, \omega_{p}$ are the associated weights given as

$$
\begin{gathered}
\omega_{q}=\frac{2^{q-1} q ! \sqrt{\pi}}{q^{2}\left[H_{q-1}\left(x_{q}\right)\right]^{2}} \\
\omega_{p}=\frac{2^{p-1} p ! \sqrt{\pi}}{q^{2}\left[H_{p-1}\left(x_{p}\right)\right]^{2}}
\end{gathered}
$$

\section{B. Kronecker Channel Model}

In this paper, we consider the correlation of antenna arrays at the receiver $(\mathrm{Rx})$ side, hence, the Kronecker channel model is applied [7]. This model allows for independent antenna array analysis at both transmitter (Tx) and Rx. We assume a MIMO communication system with a transmit array of $N_{t}$ antennas and a receive array of $N_{r}$ antennas. We can express the $N_{t} \times N_{r}$ channel matrix under the Rayleigh fading channel $\mathbf{H}$ as follows [7]

$$
\mathbf{H}=\left(\mathbf{R}_{r x}\right)^{1 / 2} \mathbf{H}_{\omega}\left\{\left(\mathbf{R}_{t x}\right)^{1 / 2}\right\}^{H}
$$


where the superscript " $\mathrm{H}$ " denotes the complex conjugate transpose, and $\mathbf{H}_{\omega}$ is a stochastic $N_{t} \times N_{r}$ matrix with (ideal independent and identical distributed) i.i.d. complex Gaussian entries with zero mean and unit variance. The $N_{r} \times N_{r}$ and $N_{t} \times N_{t}$ matrices $\mathbf{R}_{r x}$ and $\mathbf{R}_{t x}$ are the spatial correlations of MIMO antenna arrays at the $\mathrm{Rx}$ and $\mathrm{Tx}$ sides, respectively. Thus, the corresponding full channel correlation matrix $\mathbf{R}$ (in which $\otimes$ denotes the Kronecker product) is derived as

$$
\mathbf{R}=\mathbf{R}_{r x} \otimes \mathbf{R}_{t x}
$$

\section{ENERGY EFFICIENCY MAXIMIZATION}

\section{A. Energy Efficiency}

We apply the well-known definition of the systemachievable EE as the number of information bits transmitted per Joule of energy [1], i.e. $E_{\text {MIMO }}=S_{\text {MIMO }} / P_{\text {total }}$ in bits $/ \mathrm{J}$, where $S_{\text {MIMO }}$ denotes the spectral efficiency of the system, $P_{\text {total }}$ is total power of a Tx including various elements, such as the power for the baseband unit, the power amplifier, or radio-frequency circuitry in a practical setting. Thus, a power model can be given as [2]

$$
P_{\text {total }}=t\left(\xi P / t+P_{c}\right)=\xi P+t P_{c}
$$

where $P$ and $P_{c}$ are the transmit power and the loadindependent circuit power at the minimum nearly zero output power, respectively, and $\xi$ is the scaling factor of the load-dependent power. Accordingly, the EE upper bound under the Kronecker model is given by [1]

$$
\hat{E}_{\text {MIмо }}=C /\left(t P_{c}+t \xi N \exp \left\{\frac{C \ln 2}{b}+\frac{1}{b} \ln \left[\frac{(a-b) !}{a ! \operatorname{det} \mathbf{R}_{x}}\right]\right\}\right)
$$

where $a=\max \left\{N_{t}, N_{r}\right\}$ and $b=\min \left\{N_{t}, N_{r}\right\}, C$ denotes the channel capacity, $N$ is the noise variance in the Gaussian distribution. $\mathbf{R}_{x}$ refers to the spatial correlation matrix at either the Tx or Rx end. The optimum value of spectral efficiency that maximizes the EE can be also obtained from [1] as

$$
S_{\text {MIMO }}^{*}=\frac{b}{\ln (2)}+\frac{b}{\ln (2)} \omega\left\{\frac{P_{c}}{\xi N} \exp \left(-\frac{1}{b} \ln \left[\frac{(a-b) !}{a ! \operatorname{det} \mathbf{R}_{x}}\right]\right)\right\}
$$

where $\omega\{$.$\} is the Lambert \omega$ function satisfying $\omega(z) e^{\omega(z)}=z$. Note that for a system with unit bandwidth, the spectral efficiency equals to channel capacity, i.e. $S_{\text {MIMO }}=C$. Therefore, we can quote the channel capacity to replace spectral efficiency from now on. Substituting (14) into (13) with $S_{\text {MIMO }}=C$, results the upper bounded EE with optimal channel capacity, where our SCF matrix $R_{s}(m, n)$ is used instead of $\mathbf{R}_{x}$

\section{B. Particle Swarm Optimization}

PSO is widely utilized in various fields taking advantage of a swarm with multiple particles to solve multidimensional optimization problems. PSO uses the information of social interaction between independent agents and fitness to find the optimal solution. In this paper, the upper bounded EE with SCF becomes the objective function for the algorithm. The particles inside the swarm are motivated to find the locations of antenna array elements in a constrained 3D space.

The key formulas of velocity $\mathbf{v}_{i}$ and position $\mathbf{x}_{i}$ update for the $i$ th particle at each moment $t(t=0,1,2, \ldots)$ are given by [10]

$$
\begin{gathered}
\mathbf{v}_{i}(t+1)=\omega \mathbf{v}_{i}(t)+c_{1} r_{1}\left[\mathbf{p}_{i}(t)-\mathbf{x}_{i}(t)\right]+c_{2} r_{2}\left[g(t)-\mathbf{x}_{i}(t)\right] \\
\mathbf{x}_{i}(t+1)=\mathbf{x}_{i}(t)+\mathbf{v}_{i}(t+1)
\end{gathered}
$$

where $\mathbf{p}_{i}(t)$ and $g(t)$ are the personal best (pbest) position of the $i$ th particle and the global best (gbest) position respectively. $\omega \sim(0,1)$ denotes inertial weight which keeps the particle not to be affected by the pull of gbest and pbest. $c_{1}$ and $c_{2}$ are scaling factors namely acceleration coefficients for pbest and gbest respectively. $r_{1}$ and $r_{2}$ are random variables between $(0,1)$.

\section{Exponential Correlation Model for Comparison}

As widely used in literature, the exponential correlation matrix is derived by [11]

$$
\boldsymbol{\Phi}_{t r}=\left\{\begin{array}{c}
\phi^{|r-t|}, r \leq t \\
\left(\phi^{|r-t|}\right)^{*}, r>t
\end{array}\right.
$$

where the single coefficient $\phi$ satisfies $|\phi| \leq 1$. Consider $\boldsymbol{\Phi}$ as a $l \times l$ correlation matrix, thus

$$
\operatorname{det} \boldsymbol{\Phi}=(1-\phi \bar{\phi})^{l-1}=\left(1-|\phi|^{2}\right)^{l-1}
$$

where $l$ could equal either $r$ or $t$ depending on the correlation at which side. Inserting (17) into (13), results the upper bounded EE of the Rayleigh fading channel with exponential correlation to compare with the optimized EE by using our SCF in eq., (7).

\section{Simulation Results}

Inserting the optimum channel capacity of (14) into (13) and using (17), we obtain the maximum EE with exponential SCF. Without loss of generality, we set the noise variance $N$ to be unity. The parameters in the power model are $\xi=2.4, P_{c}=130$ as set in [1]. In Fig. 2, the optimal EE is plotted for increased values of the exponential correlation coefficient with various numbers of $\mathrm{Tx}$ and $\mathrm{Rx}$ array elements. It is clear from Fig. 2 that the EE decreases as spatial correlation increases, which demonstrates a clear 


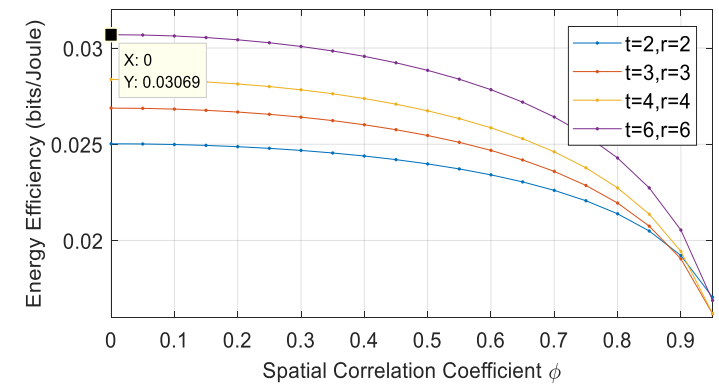

Fig. 2. EE versus spatial correlation coefficient with $t \mathrm{Tx}$ and $\mathrm{r} \mathrm{Rx}$ antennas.

potential to optimize the EE given the number of antenna array elements within a limited space.

We then employ the PSO algorithm that finds the optimal antenna array topology in space by maximizing the EE. Given the number of Tx and Rx antenna array elements, our analysis focuses on the antenna array structure at the Rx side only by using the Kronecker model [7]. The parameters used are as follow: $N_{t}=N_{r}=6, M E O A=7 \pi / 18$, $M A O A=\pi / 2, E S=\pi / 36, A S=\pi / 60$. The boundary radius of the searching space is $D_{\max }=10 \lambda$, the spatial correlation matrix for Tx antenna arrays $\mathbf{R}_{t x}$ is set to be an identity matrix. The summation terms for Hermite polynomials are $\mathrm{M}=\mathrm{N}=5$ as verified in [8]. Regarding to the algorithm, we choose 16 particles running for 500 times in the limited space for each simulation. After EE optimization by using our spatial correlation modeling approach, a six-antenna array topology at the Rx side arises as shown in Fig. 3. The corresponding optimal EE is $0.035734 \mathrm{bits} /$ Joule, which is considerably higher than the maximum EE (0.03069 bits/Joule) for six arrays at both Tx and Rx sides provided by exponential correlation model (see Fig. 2). Note that the result of the optimal EE is an average sample obtained by running Monte Caro statistics for 1000 times.

A further comparison is made between the model used in [1] and our model for optimal capacity to maximize EE. Employing eq. (13) and setting $\phi=0$ in eq. (17), as plotted in Fig. 4, the relation between EE and channel capacity for various numbers of antennas in i.i.d. channels is obtained. For $N_{t}=N_{r}=6$, the optimal channel capacity equals to $34.67 \mathrm{bits} / \mathrm{s} / \mathrm{Hz}$ and the corresponding maximum $\mathrm{EE}$ is $0.03321 \mathrm{bits} /$ Joule by using the model in [1]. Comparing to this capacity, the optimal capacity acquired from our model equals to $45.62 \mathrm{bits} / \mathrm{s} / \mathrm{Hz}$ for the maximum $\mathrm{EE}$ (0.035734 bits/Joule), which are both significantly higher. This result shows that our model can offer a wider range for the upper bound of EE and optimal channel capacity and thus, is more practical to realistic propagation environments.

\section{CONCLUSION}

We employed a flexible and generalized 3D AOA model to account for realistic propagation environments. A novel closed-form SCF relevant to the location of antenna array elements in the 3D space has been derived. Using the relationship between EE and channel capacity, the maximum EE obtained by our model was considerably higher than that of derived by the exponential SCF. Furthermore, the corresponding optimal channel capacity that maximizes the

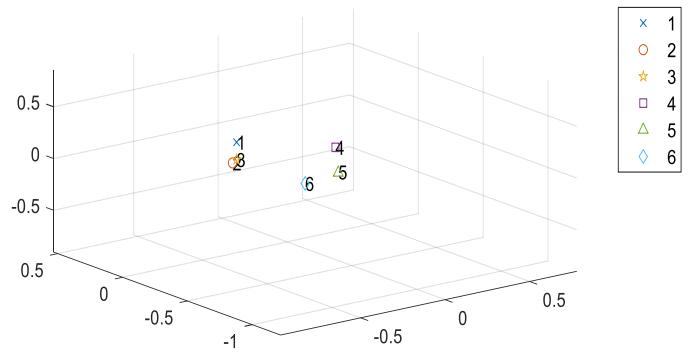

Fig. 3. A six-antenna array topology after EE optimization.

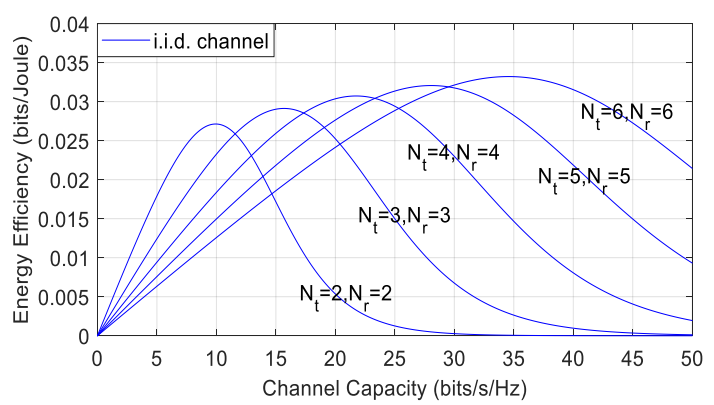

Fig. 4. EE versus channel capacity for various numbers of antennas in i.i.d. channels.

EE was also significantly higher. The generalized and flexible modeling approach proposed in this work can be used for arbitrary number of antenna elements and adapt to any realistic propagation environment, by using other AOA models. We provided new insights into EE maximization for antenna arrays, while the simulation results demonstrated the effectiveness and usefulness of the proposed research approach.

\section{REFERENCES}

[1] J. Jiang, M. Dianati, M. A. Imran, R. Tafazolli, and Y. Chen, "On the relation between energy efficiency and spectral efficiency of multipleantenna systems," IEEE Transactions on Vehicular Technology, vol. 62, no. 7, pp. 3463-3469, 2013.

[2] G. Auer, V. Giannini, I. GÓdor, O. Blume, A. Fehske, J. A. Rubio, P. Frenger, M. Olsson, D. Sabella, M. J. Gonzalez, M. A. Imran, and C. Desset, "How much energy is needed to run a wireless network?," Green Radio Communication Networks, vol. 9781107017, no. June, pp. 359-384, 2012.

[3] H. Li, L. Song, and M. Debbah, "Energy efficiency of large-scale multiple antenna systems with transmit antenna selection," IEEE Transactions on Communications, vol. 62, no. 2, pp. 638-647, 2014.

[4] O. K. Rayel, G. Brante, J. L. Rebelatto, R. D. Souza, and M. A. Imran, "Energy efficiency-spectral efficiency trade-off of transmit antenna selection," IEEE Transactions on Communications, vol. 62, no. 12, pp. 4293-4303, 2014.

[5] H. Ren, N. Liu, C. Pan, and C. He, "Energy Efficiency Optimization for MIMO Distributed Antenna Systems," IEEE Transactions on Vehicular Technology, vol. 66, no. 3, pp. 2276-2288, 2017.

[6] X. Li, X. Ge, X. Wang, J. Cheng, and V. C. M. Leung, "Energy Efficiency Optimization: Joint Antenna-Subcarrier-Power Allocation in OFDM-DASs," IEEE Transactions on Wireless Communications, vol. 15 , no. 11, pp. 7470-7483, 2016.

[7] J.-H. Lee and C.-C. Cheng, "Spatial correlation of multiple antenna arrays in wireless communication systems," Progress In Electromagnetics Research, vol. 132, pp. 347-368, 2012.

[8] L. Zhang, Z. Luo, and S. H. Leung, "An efficient approximation of spatial correlation based on Gauss-Hermite quadrature," IEEE Transactions on Signal Processing, vol. 66, no. 3, pp. 617-626, 2018. 
[9] S. K. Yong and J. S. Thompson, "Three-dimensional spatial fading correlation models for compact MIMO receivers," IEEE Transactions on Wireless Communications, vol. 4, no. 6, pp. 2856-2868, 2005.

[10] J. Robinson and Y. Rahmat-Samii, "Particle swarm optimization in electromagnetics," IEEE Transactions on Antennas and Propagation, vol. 52 , no. 2 , pp. $397-407,2004$
[11] S. Chatzinotas, M. A. Imran, and R. Hoshyar, "On the Multicell Processing Capacity of the Cellular MIMO Uplink Channel in Correlated Rayleigh Fading Environment," IEEE Transactions on Wireless Communications, vol. 8, no. 7, pp. 3704-3715, 2009. 